\title{
An Analysis of the Malassezia Species Distribution in the Skin of Patients with Pityriasis Versicolor in Chengdu, China
}

\author{
Zhen Xie, ${ }^{1,2}$ Yuping Ran, ${ }^{1}$ Hao Zhang, ${ }^{1}$ Min Zhang, ${ }^{3}$ Huiying Wan, ${ }^{1}$ and Conghui Li ${ }^{1}$ \\ ${ }^{1}$ Department of Dermatology, West China Hospital, Sichuan University, Chengdu 610041, China \\ ${ }^{2}$ Department of Dermatology, Sichuan Academy of Medical Science \& Sichuan Provincial People's Hospital, Chengdu 610072, China \\ ${ }^{3}$ Department of Gastroenterology, The Sixth Affiliated Hospital, Sun Yat-sen University, Guangzhou 510000, China
}

Correspondence should be addressed to Yuping Ran; ranyuping@vip.sina.com

Received 19 May 2014; Revised 15 July 2014; Accepted 18 July 2014; Published 10 August 2014

Academic Editor: Jean-Philippe Bouchara

Copyright (C) 2014 Zhen Xie et al. This is an open access article distributed under the Creative Commons Attribution License, which permits unrestricted use, distribution, and reproduction in any medium, provided the original work is properly cited.

\begin{abstract}
Pityriasis versicolor (PV) is a common clinical problem associated with Malassezia species (Malassezia spp.). Controversies remain regarding the specific species involved in the development of PV. This study analyzed the difference in Malassezia spp. distribution in lesional and nonlesional skin in Chinese PV patients. A paired design was applied. Lesional and nonlesional scales from 24 cases were collected; real-time fluorescence quantitative PCR was used to detect 10 different Malassezia spp. In lesional skin, the highest detection rates were for M. globosa (95.8\%), M. restricta (91.7\%), and M. sympodialis (50.0\%). In nonlesional skin, the highest detection rates were for $M$. globosa (87.5\%), M. restricta (79.2\%), and M. dermatis (33.3\%). A significant difference in the detection rate was only found for $M$. sympodialis (50.8\% versus $20.8 \%, P=0.04$ ). Compared with nonlesional skin, the amount of $M$. globosa, M. restricta, and $M$. sympodialis in lesional skin was significantly higher $(3.8 \pm 1.3,2.5 \pm 1.1$, and $3.2 \pm 1.6$ times higher, resp.). The results of this study do not indicate that $M$. globosa and $M$. restricta are directly correlated with PV development; however, $M$. sympodialis is more likely related to PV development in Chinese individuals.
\end{abstract}

\section{Introduction}

The lipid-dependent yeast Malassezia species (Malassezia sp.) is widely found on the skin surface of humans and other animals $[1,2]$. This fungus can cause pityriasis versicolor, Malassezia folliculitis, and seborrheic dermatitis. Additionally, its infection can worsen the condition of immunocompromised infants and young children [3-6].

It was recognized in 1846 that the pathogen of pityriasis versicolor (PV) was a fungus [7], but it was first named as the genus Malassezia by Baillon until 1889. By 2011, 14 different Malassezia spp. had been differentiated by morphology, biochemistry, rDNA sequencing, restriction fragment length polymorphism (RFLP), and other methods. These species include $M$. furfur, $M$. sympodialis, $M$. obtusa, . globosa, $M$. restricta, $M$. slooffiae, $M$. pachydermatis, $M$. dermatis [8], $M$. japonica [9], M. yamatoensis [10], M. nana [11], M. caprae, $M$. equina [12], and $M$. cuniculi [13]. There have been reports of $M$. pachydermatis in humans $[4,14]$, whereas $M$. nana, $M$. caprae, M. equina, and M. cuniculi are pro-animal species that have never been detected in humans. The other species exist in human skin with varying distributions across species depending on the location and the local microenvironmental variation.

To date, controversies remain over the specific species involved in the development of $\mathrm{PV}$, and the results from different countries are not consistent. In general, it is believed that M. globosa is the main species correlating with PV development, and this idea is mainly based on the analyses of the predominant species of lesional skin in the Japanese population $[15,16]$. In other districts and regions, such as Greece and Spain, similar reports have also been found [17, 18]. In some studies that analyzed species differences between lesional and nonlesional skin, the detection rate of M. globosa in lesional skin was higher compared with nonlesional skin $[17,19]$. However, there was also Malassezia spp. distribution in nonlesional skin of healthy individuals and PV patients, with extremely high detection rates in some studies; for example, the detection rate of Malassezia in healthy Japanese 
individuals was as high as $86.7 \%$ [20]. Moreover, Miranda et al. showed that $M$. furfur was the predominant species in the lesional skin of PV patients in Brazil [21], whereas Gupta et al. showed in a Canadian population and Ramadán et al. showed in Argentineans that $M$. sympodialis was more common in PV patients $[22,23]$. In Gupta's study, the detection rate of $M$. globosa in lesional skin was only $6.3 \%$ [22]. The differences in these studies might be related to the regional differences of Malassezia distribution [24-26]. Although PV is common in China [27], there have not been reports on Malassezia spp. distribution in the Chinese PV population to date.

Based on these findings, we conducted the current study to analyze the constituents of Malassezia spp. in lesional skin and nonlesional skin in Chinese PV patients using realtime fluorescence quantitative polymerase chain reaction (RT-PCR). The results of this study may shed light on the pathogenesis of $\mathrm{PV}$.

\section{Materials and Methods}

2.1. Study Subjects. Twenty-four patients with PV from an outpatient clinic consecutively entered this study from April 2010 to July 2010. PV was diagnosed by its clinical appearance and the staining of scales with $20 \% \mathrm{KOH}$ and Parker blue ink in microscopic examination. The inclusion and exclusion criteria included the following: (1) typical clinical manifestations: pigmentation or hypopigmentation spots on the body trunk or the forearm; (2) lesional scales that showed thick and short hyphae and round or oval spores under the microscope with methylene blue staining; (3) no antifungal agents administered orally or externally within one month before the visit; (4) no Malassezia-related diseases, such as Malassezia folliculitis, atopic dermatitis, or seborrheic dermatitis. The clinical diagnoses of the patients were made by the same dermatologist.

All enrolled patients signed an informed consent form. All procedures were in accordance with the Declaration of Helsinki and were approved by the Ethics Committee of the Sichuan Academy of Medical Science \& the Sichuan Provincial People's Hospital, China.

\subsection{Scale Sample Collection and Genome DNA Extraction.} For each patient, 1 lesional scale sample and 1 nonlesional scale sample were collected. The samples were collected using a previously published method [28]. Malassezia samples were collected by applying Tegaderm transparent dressings $(6 \times$ $7 \mathrm{~cm}$; $3 \mathrm{M}$ Health Care, Neuss, Germany) on the target skin of patients with PV. The collected transparent dressings were placed in a $1 \%$ Triton X-100 solution, and the suspensions were transferred into sterile Eppendorf tubes. The supernatants were removed by centrifugation at $4,000 \times \mathrm{g}$. The sample collection was conducted by the same dermatologist, and the lesional scale samples were obtained from thoracic lesions to reduce the impact of the location on the distributions of the species. Malassezia spp. are lipid-dependent yeasts that mainly colonize skin that easily develops seborrhea, such as the chest, back, and face. We collected samples of thoracic lesions and of distant nonlesional skin from the same PV
TABLE 1: Universal fungal primer PCR reaction system.

\begin{tabular}{lc}
\hline Components & Sample amount \\
\hline & $12.5 \mu \mathrm{L}$ (including 2.5 $\mathrm{U}$ Pfu Taq enzyme, \\
PCR reaction mixture & $500 \mu \mathrm{M}$ dNTP, 20 mM Tris-Cl $(\mathrm{pH} \mathrm{8.3),}$ \\
& $100 \mathrm{mM} \mathrm{KCl}$, and $\left.3 \mathrm{mM} \mathrm{MgCl}_{2}\right)$ \\
ITS1 $^{\mathrm{a}}$ & $1 \mu \mathrm{L}$ \\
ITS4 $^{\mathrm{b}}$ & $1 \mu \mathrm{L}$ \\
Template DNA & $2 \mu \mathrm{L}$ \\
Double distilled water & $8.5 \mu \mathrm{L}$ \\
\hline
\end{tabular}

${ }^{\mathrm{a}}$ Internal transcribed spacer $1 ;{ }^{\mathrm{b}}$ internal transcribed spacer 4 .

patient and performed comparisons to reduce the influence of the skin microenvironment of different individuals on Malassezia colonization. Because Malassezia hyphae are found on skin near PV skin lesions [19], skin distant from the lesions was selected as the area of nonlesional skin sampling in this study. In these areas, hyphae were not detected. A total of 10 Malassezia reference strains that are maintained in our laboratory were utilized as positive controls [28]. Sampling and DNA extraction followed methods previously described in the literature [28] and the OMEGA D3370-01 kit instructions.

2.3. Verification of DNA Extraction through PCR with Universal Fungal Primers. The primers were universal fungal primers: ITS1 ( $5^{\prime}$-TCCGTAGGTGAACCTGCG-3 $\left.{ }^{\prime}\right)$ and ITS4 $\left(5^{\prime}\right.$-TCCTCCGCTTATTGATATGC- $\left.3^{\prime}\right)$. The constituents of the reaction system and the total volumes are summarized in Table 1. PCR, electrophoresis, and other laboratory tests were all performed using a blind method.

The reaction conditions consisted of predenaturation for $5 \mathrm{~min}$ at $94^{\circ} \mathrm{C}, 35$ cycles of $94^{\circ} \mathrm{C}$ for $45 \mathrm{~s}, 58^{\circ} \mathrm{C}$ for $45 \mathrm{~s}$, and $72^{\circ} \mathrm{C}$ for $45 \mathrm{~s}$, and a final step at $72^{\circ} \mathrm{C}$ for $7 \mathrm{~min}$. The PCR products were processed by $1.5 \%$ agarose electrophoresis for $35 \mathrm{~min}$, and the results were observed under UV light and photographed. A negative control was tested during each amplification. The amplified products were considered valid only when there was no DNA band generated after the amplification of the negative control.

2.4. Extracted DNA Concentration as Determined by Spectrophotometry. Extracted DNA concentrations from the lesional and nonlesional skin were determined by spectrophotometry to determine whether there were different concentrations in the lesion and nonlesion groups and to ensure that the initial concentrations of the templates for real-time fluorescence quantitative PCR were the same. If the initial concentrations of the two groups were not the same, corrections were made by dilution. The TE solution that contained dissolved DNA was set as the blank control. One microliter of sample was drawn by a micropipette and dropped onto the test platform, and the absorbance and concentration of the sample were determined $(\mu \mathrm{g} / \mu \mathrm{L})$.

2.5. RT-PCR. The design of the primers and the probes was based predominately on the ITS sequences of 10 Malassezia 
TABLE 2: Primers and probes used in RT-PCR.

\begin{tabular}{|c|c|c|c|}
\hline Species & Sense primer $\left(5^{\prime} \rightarrow 3^{\prime}\right)$ & Antisense primer $\left(5^{\prime} \rightarrow 3^{\prime}\right)$ & TaqMan probe \\
\hline Malassezia & GTAGACTCCATCTAAAGCTAAAT & СТTTTAACTCTСТTTCСAAAGT & CCCTCACGGTACTTGTTCGCT \\
\hline M. furfur & GTGAATTGCAGAATTCCGTGAAT & GAGCCTGTTTCTTGCGAAACA & CTTTGAACGCACCTTGCGCTC \\
\hline M. globosa & GTGAATTGCAGAATTCCGTGAAT & GAGCTTTTTCTAGAGAAGAAAAG & CTTTGAACGCACCTTGCGCTC \\
\hline M. obtusa & GTGAATTGCAGAATTCCGTGAAT & GCGAGCCTGTTTAGCAAGAAA & CTTTGAACGCACCTTGCGCTC \\
\hline M. pachydermatis & GTGAATTGCAGAATTCCGTGAAT & GAGCCTGTAGTTTCCCACAG & CTTTGAACGCACCTTGCGCTC \\
\hline M. restricta & GTGAATTGCAGAATTCCGTGAAT & GCGAGCCTGTGCTAGGTA & CTTTGAACGCACCTTGCGCTC \\
\hline M. slooffiae & GTGAATTGCAGAATTCCGTGAAT & СTTTTCGAGCGAGCCTACCAA & CTTTGAACGCACCTTGCGCTC \\
\hline M. sympodialis & GTGAATTGCAGAATTCCGTGAAT & TACAATCCCCAGGCAGCAA & СTTTGAACGCACCTTGCGCTC \\
\hline M. yamatoensis & GTGAATTGCAGAATTCCGTGAAT & GCCAGCCTCGCAAGGCAT & CTTTGAACGCACCTTGCGCTC \\
\hline M. japonica & GTGAATTGCAGAATTCCGTGAAT & TGTACGAGACACTGGCAGGCA & CTTTGAACGCACCTTGCGCTC \\
\hline M. dermatis & GTGAATTGCAGAATTCCGTGAAT & GTTTCCCAGGCAGCGGCA & CTTTGAACGCACCTTGCGCTC \\
\hline
\end{tabular}

TABLE 3: Reaction system for real-time fluorescence quantitative PCR.

\begin{tabular}{lc}
\hline Components & Sample amount \\
\hline $10 \times$ buffer $\left(\right.$ without $\left.\mathrm{Mg}^{2+}\right)$ & $3 \mu \mathrm{L}$ \\
$\mathrm{MgCl}_{2}(25 \mathrm{mM})$ & $3 \mu \mathrm{L}$ \\
$\mathrm{dNTP}(25 \mathrm{mM})$ & $0.36 \mu \mathrm{L}$ \\
Sense prime $(10 \mu \mathrm{M})$ & $1 \mu \mathrm{L}$ \\
Antisense primer $(10 \mu \mathrm{M})$ & $1 \mu \mathrm{L}$ \\
TaqMan probe $(10 \mu \mathrm{M})$ & $0.6 \mu \mathrm{L}$ \\
Taq enzyme $(5 \mathrm{U} / \mu \mathrm{L})$ & $0.3 \mu \mathrm{L}$ \\
Double distilled water & $18.74 \mu \mathrm{L}$ \\
Template DNA & $2 \mu \mathrm{L}$ \\
\hline
\end{tabular}

species released by GenBank. Primer 5.0 software was used to design the Malassezia-specific primers, the species-specific primers, and their corresponding TaqMan fluorescent probes to amplify the Malassezia species and the 10 different Malassezia target DNAs. The specific sequences are shown in Table 2. The 48 DNA samples underwent fluorescence quantitative PCR and reacted with the Malassezia genus-specific primer/probe and the 10 sets of Malassezia primers/probes. The qualitative results (positive bands) and quantitative results (Ct values) were recorded.

The reaction was carried out in a $0.2 \mathrm{~mL}$ EP tube in $30 \mu \mathrm{L}$ of reaction volume. The components are summarized in Table 3. The PCR conditions consisted of a predenaturation step at $94^{\circ} \mathrm{C}$ for $2 \mathrm{~min}, 45 \mathrm{cycles}$ of $94^{\circ} \mathrm{C}$ for $20 \mathrm{~s}, 50^{\circ} \mathrm{C}$ for $20 \mathrm{~s}$, and $60^{\circ} \mathrm{C}$ for $30 \mathrm{~s}$, and a final extension step at $72^{\circ} \mathrm{C}$ for $7 \mathrm{~min}$.

The amplification cycle number when the fluorescence intensity reached a certain threshold (Ct value) in each sample tube was determined according to the kinetic curve. Water was used as the template and the negative control during each amplification. The amplified products were considered valid only when there was no DNA band generated after the amplification of the negative control.

2.6. Specific Verification Using Malassezia Genus-Specific Primers and Species-Specific Primers. Malassezia reference strains were used as the positive controls, and fluorescence quantitative PCR products were collected. The products
TABle 4: Detection rates of multiple Malassezia spp. in the same sampling area (\%).

\begin{tabular}{lccc}
\hline & $\begin{array}{c}\text { 2 Malassezia } \\
\text { spp. detected }\end{array}$ & $\begin{array}{c}\text { 3 Malassezia } \\
\text { spp. detected }\end{array}$ & $\begin{array}{c}\text { 4 Malassezia } \\
\text { spp. detected }\end{array}$ \\
\hline Nonlesional skin & 8.3 & 41.7 & 50.0 \\
Lesional skin & 16.7 & 50.0 & 33.3 \\
\hline
\end{tabular}

were sent to Shanghai Biological Engineering Co., Ltd., with their corresponding primers to be sequenced for verification. The results were compared with the sequences from GenBank (http://blast.st-va.ncbi.nlm.nih.gov/Blast.cgi), and the specificity of the primers was determined based on this comparison.

2.7. Statistical Analysis. SPSS13.0 (Chicago, IL, USA) was utilized in the statistical analyses. Data with normal distributions are presented as the $\bar{X} \pm S$ (mean \pm standard deviation), and the enumeration data are expressed as percentages. A $\chi^{2}$ test was used in the comparison of species detection rates of lesional skin and nonlesional skin, and a $t$-test was used in the quantitative comparison of species in lesional skin and nonlesional skin; a two-tailed $P$ value $<0.05$ was regarded as a statistically significant difference.

\section{Results}

3.1. ITS Amplification Results. The ITS1/ITS4 amplification of the lesional and nonlesional skin scale DNA samples from patients 1-24 is shown in Figure 1. All 48 samples from 24 patients in this study demonstrated positive bands at 600800 bp after ITS1/ITS 4 universal fungal primer amplification.

\subsection{Malassezia Species Distribution}

3.2.1. Malassezia Species in Lesional and Nonlesional Skin. First, we determined whether more Malassezia spp. were detected in lesions than in nonlesional skin. The detection rates of Malassezia spp. in lesional and nonlesional skin from the same sampling area are shown in Table 4; the detection rates were similar $(3.2 \pm 0.7$ versus $3.4 \pm 0.7, P=0.208)$, 


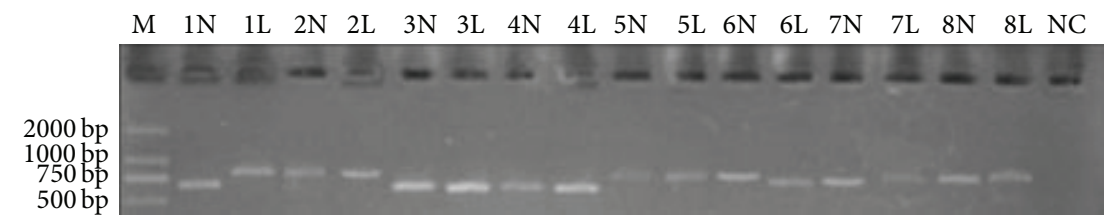

FIGURE 1: Electropherogram of the lesional and nonlesional skin DNA samples from patients 1-8 after ITS1/ITS4 amplification. The lanes of the lesional scales are coded as the patient number $+\mathrm{L}$ (lesion), and the lanes of the nonlesional skin samples are coded as the patient number $+\mathrm{N}$ (normal). M signifies the marker, and NC signifies the negative control. Positive bands appeared at the 600-800 bp position in each sample lane. No band appeared in the NC.

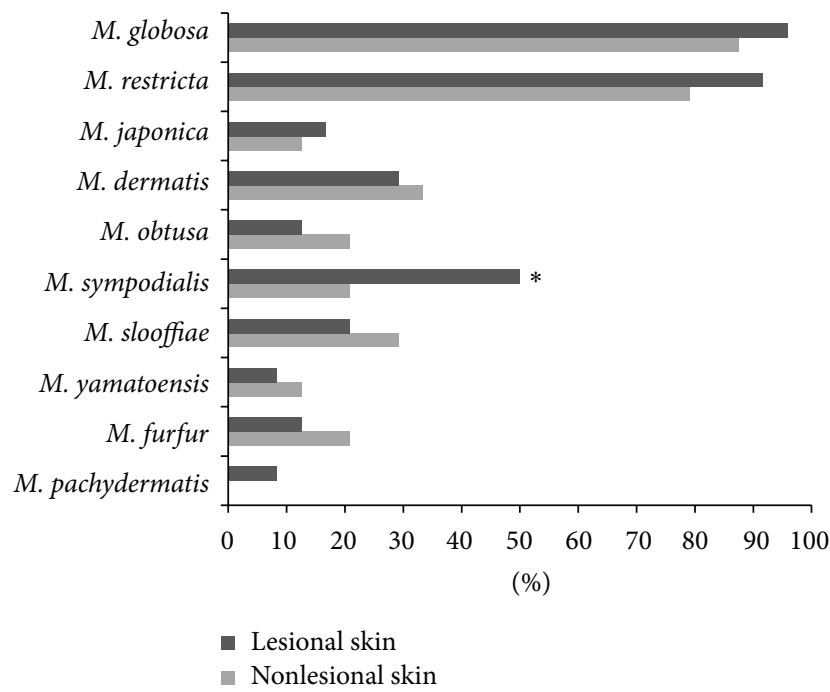

Figure 2: Malassezia species detection rates in the lesional and nonlesional skin of the 24 patients. ${ }^{*} P<0.05$, nonlesional skin versus lesional skin.

indicating that the number of Malassezia species detected did not correlate with the development of PV.

The detection rate of each Malassezia species is shown in Figure 2. Ten Malassezia species were detected in the lesional skin, and 9 Malassezia species were detected in the nonlesional skin of the 24 patients with PV. M. globosa possessed the highest detection rates in both the lesional and nonlesional skin, which were $95.8 \%$ and $87.5 \%$, respectively. Qualitatively, M. globosa had the highest detection rate, followed by $M$. restricta, with rates of $91.7 \%$ and $79.17 \%$, respectively. In the lesional skin, 10 species of Malassezia were detected, while $M$. pachydermatis was not detected in the nonlesional skin. In the lesional skin, the first three species of the highest detection rates were M. globosa (95.8\%), $M$. restricta (91.7\%), and M. sympodialis (50.0\%), respectively. In the nonlesional skin, the first three species of the highest detection rates were $M$. globosa (87.5\%), M. restricta (79.2\%), and $M$. dermatis (33.3\%), respectively. Among the 9 species detected in both the lesional and the nonlesional skin, a significant difference in the detection rate was only found in M. sympodialis ( $50.8 \%$ versus $20.8 \%, P=0.04$ ) (Figure 2 ). The compositions of the mixed Malassezia spp. detected in the same area were mainly $M$. globosa with $M$. restricta and M. globosa with M. sympodialis. Compared with nonlesional

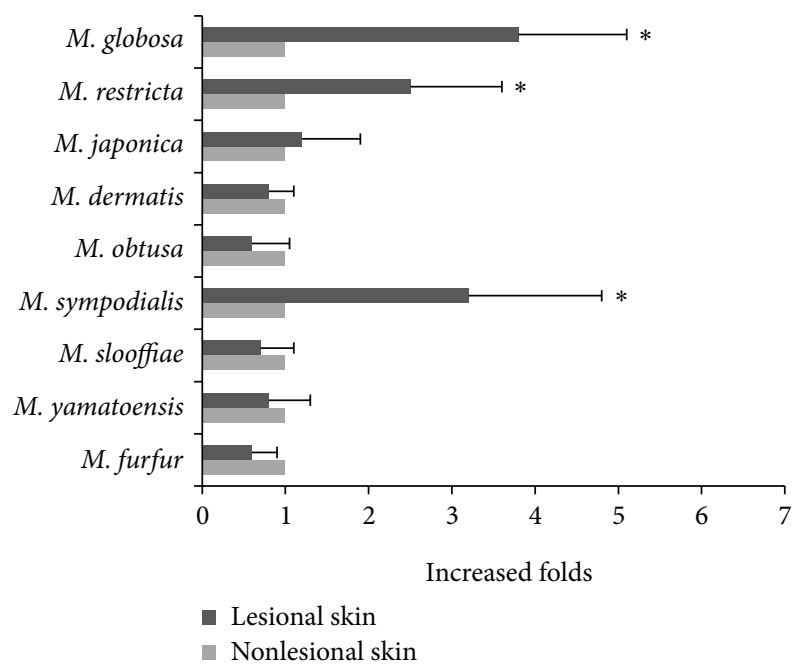

Figure 3: The relative quantity of each Malassezia species in the lesions compared with the nonlesional skin of the 24 patients. The $y$-axis displays the increased prevalence of the species in the lesions compared with the nonlesional skin. ${ }^{*} P<0.05$, nonlesional skin versus lesional skin.

skin, the rates of simultaneously detecting $M$. globosa with $M$. restricta (91.7\% versus $66.7 \%, P=0.033$ ) and $M$. globosa with M. sympodialis $(4.2 \%$ versus $50 \%, P<0.001)$ were higher in lesional skin, whereas there was no difference in the rates of simultaneously detecting M. globosa with the other 6 species in lesional versus nonlesional skin.

3.2.2. Quantitative Analysis of Malassezia Species in Lesional and Nonlesional Skin. Lesional and nonlesional skin scale samples from the 24 patients with PV were amplified by fluorescence quantitative PCR using Malassezia genus-specific primers and 10 species-specific primers. The quantity of M. globosa, M. restricta, and M. sympodialis in the lesions was significantly higher compared with nonlesional skin (Figure 3); there was no significant difference in the quantity of the other Malassezia spp. between the groups.

\section{Discussion}

$\mathrm{PV}$ is a relatively common clinical condition, which is associated with the Malassezia spp. However, the specific species of 
the fungus involved in the pathogenesis of PV remains controversial. $M$. globosa and $M$. restricta are the predominant Malassezia spp. in PV patients; however, the Malassezia spp. distribution in the Chinese population remains unclear. In this study, we analyzed the distribution of the Malassezia spp. in both lesional and nonlesional regions of the Chinese PV patients.

Malassezia sp. is a global resident flora on the skin surfaces of humans and animals, which can cause PV, Malassezia folliculitis, and seborrheic dermatitis, and its complicated infection can worsen the condition of immunocompromised infants and young children [4-6]. Previously, a culture method was primarily adopted to investigate the distribution of Malassezia spp.; unfortunately, this method is easily affected by the variation of microbial growth rates and culture media. In recent years, transparent dressings have been used to make scales stick to them, and DNA extraction from the scales for real-time fluorescence quantitative PCR has become a common technique to investigate Malassezia rapidly and efficiently [28, 29]. Using these methods, 14 Malassezia spp. have been identified to date, with 9 species predominately existing on human skin. Among the other 5 species that exist on animal skins, $M$. pachydermatis is predominately found in animals but can be transmitted to humans by contact with pets. Although the Malassezia spp. have different sensitivities to antifungal agents [30, 31], it is impossible to select highly sensitive medications to treat PV because of the incomplete understanding of the pathogenic species. The recurrence of $\mathrm{PV}$ remains a problem in clinical practice [24]; therefore, the identification of pathogenic species is helpful for selecting sensitive drugs in the clinic.

This is the first study to analyze the Malassezia spp. constituents in Chengdu PV patients. In this study, we used culture-independent real-time fluorescence quantitative PCR to successfully isolate the 10 Malassezia spp. that exist in humans. Our results showed that, among the 10 detected species, M. sympodialis, with the third highest detection rate, was the only species that had a significantly higher detection rate and a larger quantity in the lesions compared with nonlesional skin. This is consistent with the findings by Crespo Erchiga et al. in Canada regarding PV patients [17]. $M$. globosa and $M$. restricta are the two species with the highest detection rates $[17,32,33]$, although there was no difference in their detection rates in lesions versus nonlesional skin (Figure 2): the quantity of these species was higher in lesions than in nonlesional skin (Figure 3). Multiple Malassezia spp. were detected in the same sampling area (Table 4), but due to lack of previously published results on the number of detected Malassezia spp. we cannot determine whether this is a characteristic distribution of Malassezia spp. in Chinese PV patients; this finding may relate to the use of highly sensitive PCR in this study. Therefore, it is possible that multiple coexistent Malassezia spp. synergize to induce PV. We analyzed the differences in the coexistence of two species in lesions versus nonlesional skin and determined that the coexistence of $M$. globosa with $M$. restricta and of $M$. globosa with $M$. sympodialis was more common in lesions than in nonlesional skin, which supported the above hypothesis.
There are some limitations to this study. We did not include healthy people as blank controls for the consideration of the possible influence of individual idiosyncrasy on the results obtained in this study. The subjects in this study only represent the population in certain Chinese cities; because regional factors may influence the distribution of Malassezia spp. [24-26], our conclusions cannot be extrapolated to other regions. The relatively small sample size is another limitation of this study; because there are numerous combinations of more than two coexisting species, the number of samples in each combination is insufficient for further statistical analysis. Therefore, a larger sample size is necessary for future studies. However, as shown in Figure 2, the detection rate of other species was low, with the exception of M. globosa, $M$. restricta, and $M$. sympodialis. Therefore, we presume that the less common Malassezia spp. are not significant for the development of PV.

In summary, our findings suggest that M. sympodialis might be involved in the development of PV in China, and its mechanism of action deserves further study. Furthermore, mixed infections, such as $M$. globosa and M. restricta or $M$. sympodialis, might be involved in the pathogenesis of PV.

\section{Conflict of Interests}

The authors have no conflict of interests to declare.

\section{Acknowledgments}

This work is supported by Specialized Research Fund for the Doctoral Program of Higher Education (20050610058) and partly by Project 30570095 of National Natural Science Foundation of China.

\section{References}

[1] R. F. Gandra, W. Gambale, R. De Cássia Garcia Simão et al., "Malassezia spp. in acoustic meatus of bats (Molossus molossus) of the Amazon region, Brazil," Mycopathologia, vol. 165, no. 1, pp. 21-26, 2008.

[2] X. Lai, L. Cao, H. Tan, S. Fang, Y. Huang, and S. Zhou, "Fungal communities from methane hydrate-bearing deep-sea marine sediments in South China Sea," ISME Journal, vol. 1, no. 8, pp. 756-762, 2007.

[3] W. M. Dankner, S. A. Spector, J. Fierer, and C. E. Davis, "Malassezia fungemia in neonates and adults: complication of hyperalimentation.," Reviews of Infectious Diseases, vol. 9, no. 4, pp. 743-753, 1987.

[4] M. Larocco, A. Dorenbaum, A. Robinson, and L. K. Pickering, "Recovery of Malassezia pachydermatis from eight infants in a neonatal intensive care nursery," The Pediatric Infectious Disease Journal, vol. 7, no. 6, pp. 398-401, 1988.

[5] P. A. Mickelsen, M. C. Viano-Paulson, D. A. Stevens, and P. S. Diaz, "Clinical and microbiological featurs of infection with Malassezia pachydermatis in high-risk infants," Journal of Infectious Diseases, vol. 157, no. 6, pp. 1163-1168, 1988.

[6] J. Sizun, A. Karangwa, J. D. Giroux et al., "Malassezia furfurrelated colonization and infection of central venous catheters," Intensive Care Medicine, vol. 20, no. 7, pp. 496-499, 1994. 
[7] E. Eichstedt, "Pilzbildung in der pityriasis versicolor," Froriep Neue Notiz Natur Heilk, vol. 39, p. 270, 1846.

[8] T. Sugita, M. Takashima, T. Shinoda et al., "New yeast species, Malassezia dermatis, isolated from patients with atopic dermatitis," Journal of Clinical Microbiology, vol. 40, no. 4, pp. 1363-1367, 2002.

[9] T. Sugita, M. Takashima, M. Kodama, R. Tsuboi, and A. Nishikawa, "Description of a new yeast species, Malassezia japonica, and its detection in patients with atopic dermatitis and healthy subjects," Journal of Clinical Microbiology, vol. 41, no. 10, pp. 4695-4699, 2003.

[10] T. Sugita, M. Tajima, M. Takashima et al., "A new yeast, Malassezia yamatoensis, isolated from a patient with seborrheic dermatitis, and its distribution in patients and healthy subjects," Microbiology and Immunology, vol. 48, no. 8, pp. 579-583, 2004.

[11] A. Hirai, R. Kano, K. Makimura et al., "Malassezia nana sp. nov., a novel lipid-dependent yeast species isolated from animals," International Journal of Systematic and Evolutionary Microbiology, vol. 54, no. 2, pp. 623-627, 2004.

[12] F. J. Cabañes, B. Theelen, G. Castellá, and T. Boekhout, “Two new lipid-dependent Malassezia species from domestic animals," FEMS Yeast Research, vol. 7, no. 6, pp. 1064-1076, 2007.

[13] F. J. Cabaes, S. Vega, and G. Castell, "Malassezia cuniculi sp. nov., a novel yeast species isolated from rabbit skin," Medical Mycology, vol. 49, no. 1, pp. 40-48, 2010.

[14] D. O. Morris, K. O’Shea, F. S. Shofer, and S. Rankin, "Malassezia pachydermatis carriage in dog owners," Emerging Infectious Diseases, vol. 11, no. 1, pp. 83-88, 2005.

[15] N. Morishita, Y. Sei, and T. Sugita, "Molecular analysis of Malassezia microflora from patients with pityriasis versicolor," Mycopathologia, vol. 161, no. 2, pp. 61-65, 2006.

[16] A. Nakabayashi, Y. Sei, and J. Guillot, "Identification of Malassezia species isolated from patients with seborrhoeic dermatitis, atopic dermatitis, pityriasis versicolor and normal subjects," Medical Mycology, vol. 38, no. 5, pp. 337-341, 2000.

[17] V. Crespo Erchiga, A. Ojeda Martos, A. Vera Casano, A. Crespo Erchiga, and F. Sanchez Fajardo, "Malassezia globosa as the causative agent of pityriasis versicolor," British Journal of Dermatology, vol. 143, no. 4, pp. 799-803, 2000.

[18] G. Gaitanis, A. Velegraki, E. C. Alexopoulos, V. Chasapi, A. Tsigonia, and A. Katsambas, "Distribution of Malassezia species in pityriasis versicolor and seborrhoeic dermatitis in Greece. Typing of the major pityriasis versicolor isolate M. globosa," British Journal of Dermatology, vol. 154, no. 5, pp. 854-859, 2006.

[19] A. Prohic and L. Ozegovic, "Malassezia species isolated from lesional and non-lesional skin in patients with pityriasis versicolor," Mycoses, vol. 50, no. 1, pp. 58-63, 2007.

[20] M. Tajima, T. Sugita, A. Nishikawa, and R. Tsuboi, "Molecular analysis of Malassezia microflora in seborrheic dermatitis patients: Comparison with other diseases and healthy subjects," Journal of Investigative Dermatology, vol. 128, no. 2, pp. 345-351, 2008.

[21] K. C. Miranda, C. R. de Araujo, A. J. Soares et al., "Identification of Malassezia species in patients with pityriasis versicolor in Goiania-GO," Revista da Sociedade Brasileira de Medicina Tropical, vol. 39, pp. 582-583, 2006.

[22] A. K. Gupta, Y. Kohli, R. C. Summerbell, and J. Faergemann, "Quantitative culture of Malassezia species from different body sites of individuals with or without dermatoses," Medical Mycology, vol. 39, no. 3, pp. 243-251, 2001.
[23] S. Ramadán, M. Sortino, L. Bulacio, M. L. Marozzi, C. López, and L. Ramos, "Prevalence of Malassezia species in patients with pityriasis versicolor in Rosario, Argentina," Revista Iberoamericana de Micologia, vol. 29, no. 1, pp. 14-19, 2011.

[24] H. R. Ashbee, "Update on the genus Malassezia," Medical Mycology, vol. 45, no. 4, pp. 287-303, 2007.

[25] G. Gaitanis, A. Velegraki, E. C. Alexopoulos et al., "Malassezia furfur fingerprints as possible markers for human phylogeography," ISME Journal, vol. 3, no. 4, pp. 498-502, 2009.

[26] J. Xu, C. W. Saunders, P. Hu et al., "Dandruff-associated Malassezia genomes reveal convergent and divergent virulence traits shared with plant and human fungal pathogens," Proceedings of the National Academy of Sciences of the United States of America, vol. 104, no. 47, pp. 18730-18735, 2007.

[27] S. M. He, W. D. Du, S. Yang et al., "The genetic epidemiology of tinea versicolor in China," Mycoses, vol. 51, no. 1, pp. 55-62, 2008.

[28] H. Zhang, Y. Ran, Z. Xie, and R. Zhang, "Identification of Malassezia species in patients with seborrheic dermatitis in China," Mycopathologia, vol. 175, no. 1-2, pp. 83-89, 2013.

[29] T. Sugita, M. Tajima, H. Tsubuku, R. Tsuboi, and A. Nishikawa, "Quantitative analysis of cutaneous Malassezia in atopic dermatitis patients using real-time PCR," Microbiology and Immunology, vol. 50, no. 7, pp. 549-552, 2006.

[30] A. K. Gupta, Y. Kohli, A. Li, J. Faergemann, and R. C. Summerbell, "In vitro susceptibility of the seven Malassezia species to ketoconazole, voriconazole, itraconazole and terbinafine," British Journal of Dermatology, vol. 142, no. 4, pp. 758-765, 2000.

[31] A. Velegraki, E. C. Alexopoulos, S. Kritikou, and G. Gaitanis, "Use of fatty acid RPMI 1640 media for testing susceptibilities of eight Malassezia species to the new triazole posaconazole and to six established antifungal agents by a modified NCCLS M27-A2 microdilution method and Etest," Journal of Clinical Microbiology, vol. 42, no. 8, pp. 3589-3593, 2004.

[32] C. Aspiroz, M. Ara, M. Varea, A. Rezusta, and C. Rubio, "Isolation of Malassezia globosa and M. sympodialis from patients with pityriasis versicolor in Spain," Mycopathologia, vol. 154, no. 3, pp. 111-117, 2002.

[33] N. Morishita, Y. Sei, and T. Sugita, "Molecular analysis of Malassezia microflora from patients with pityriasis versicolor," Mycopathologia, vol. 161, no. 2, pp. 61-65, 2006. 


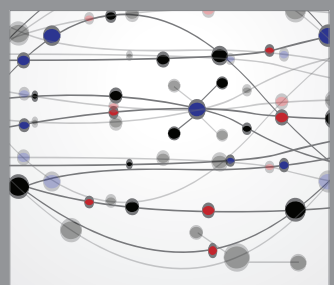

The Scientific World Journal
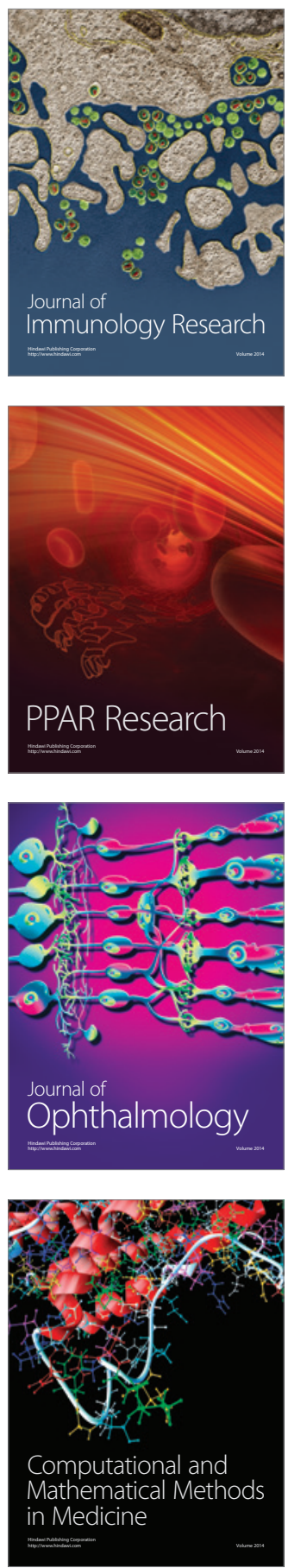

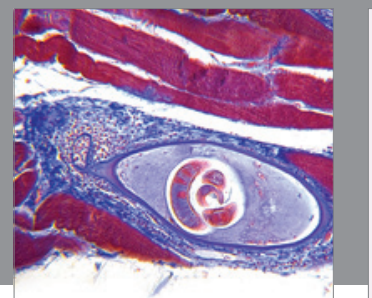

Gastroenterology

Research and Practice
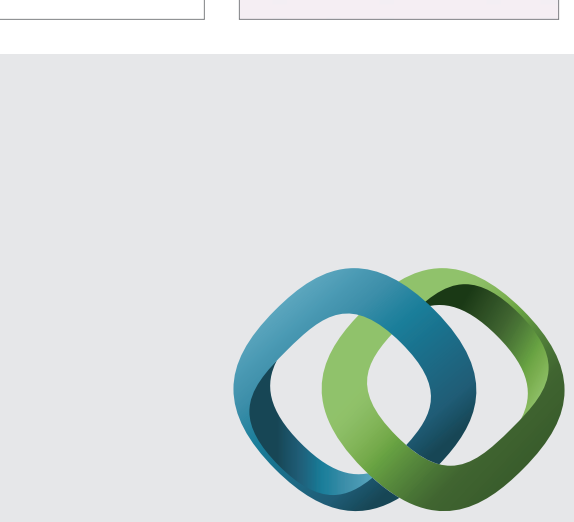

\section{Hindawi}

Submit your manuscripts at

http://www.hindawi.com
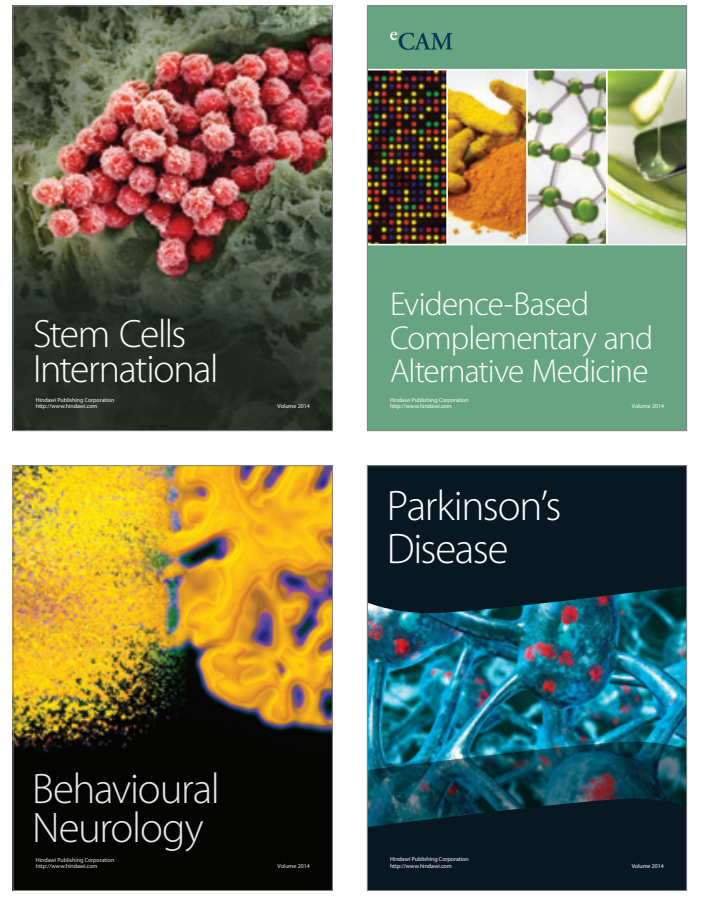
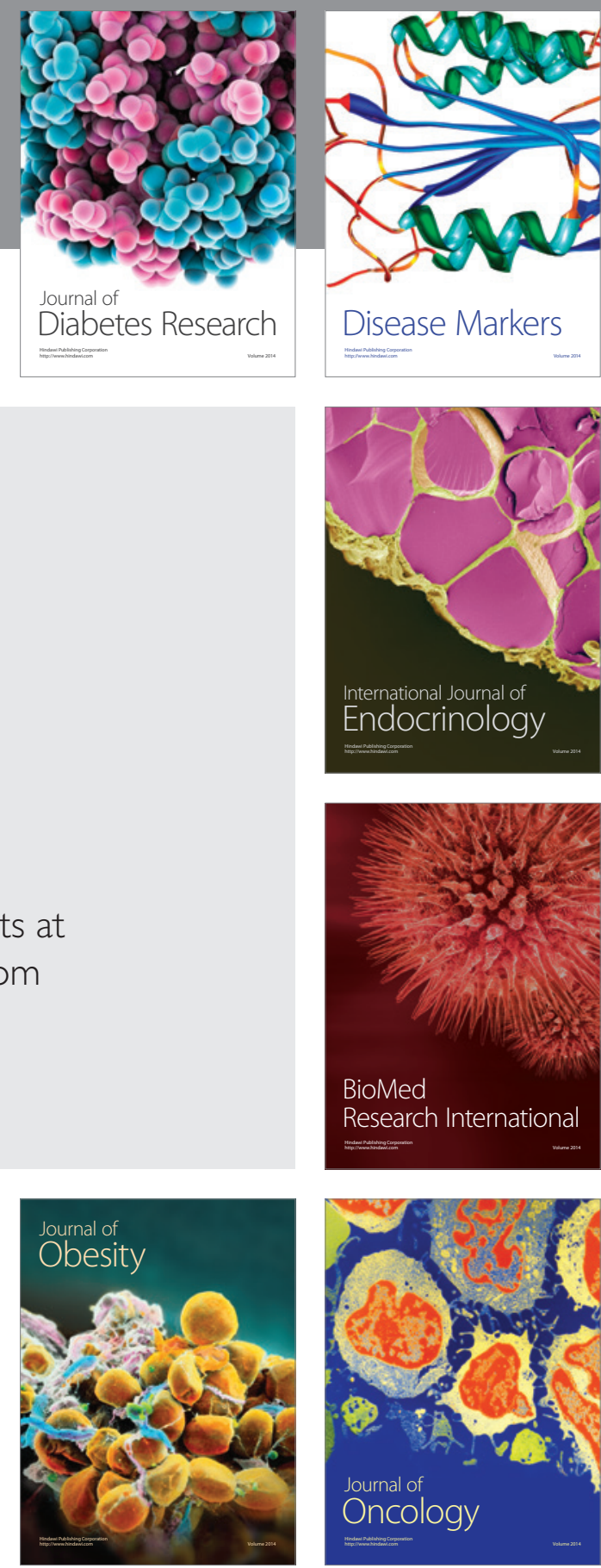

Disease Markers
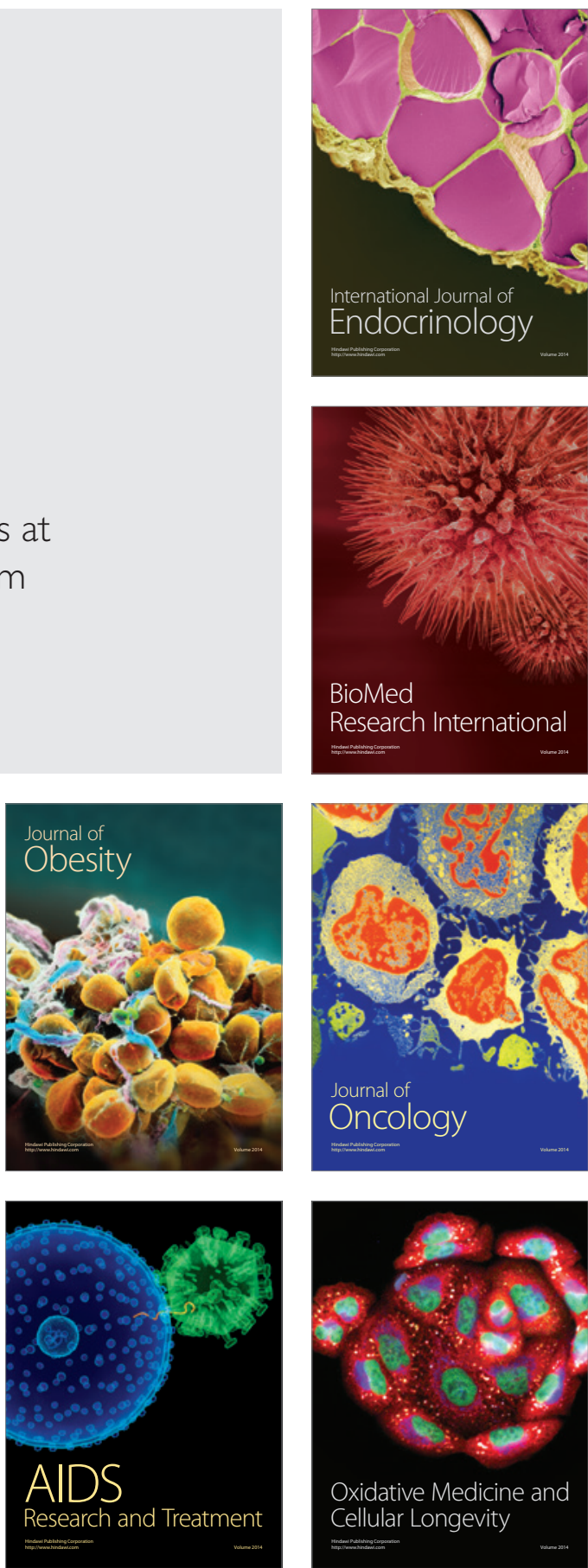